\title{
Commentary
}

This commentary provides an update for readers on the new SQUIRE 2.0 guidelines for reporting quality improvement studies.

\section{SQUIRE 2.0 (Standards for QUality Improvement Reporting Excellence) Revised Publication Guidelines From a Detailed Consensus Process}

\author{
Greg Ogrinc, MD, MS; Louise Davies, MD, MS; \\ Daisy Goodman, DNP, MPH; Paul Batalden, MD; \\ Frank Davidoff, MD; David Stevens, MD
}

\section{Autbor Affiliations: Geisel School of Medicine at Dartmouth, Hanover, New Hampshire (Drs Ogrinc, Davies, Goodman, and Batalden); Dartmouth Institute for Health Policy and Clinical Practice, Hanover, New Hampshire (Drs Ogrinc, Davies, Goodman, Batalden, Davidoff, and Stevens); Department of Veterans Affairs Medical Center, White River Junction, Vermont (Drs Ogrinc, Davies, and Goodman); and Institute for Healthcare Improvement, Cambridge, Massachusetts (Dr Stevens). Dr Davidoff is Editor Emeritus of Annals of Internal Medicine. Dr Stevens is Editor Emeritus of BMJ Quality \& Safety.}

This material is based on work supported by the Health Foundation and the Robert Wood Jobnson Foundation and included the use of facilities and material at the White River Junction VA in White River Junction, Vermont.

This is an open-access article distributed under the terms of the Creative Commons AttributionNonCommercial-NoDerivatives License 4.0 (CCBY-NCND), where it is permissible to download and share the work provided it is properly cited. The work cannot be changed in any way or used commercially.

The authors declare no conflict of interest.

This article is copublished by BMJ Quality \& Safety, BMJ Publishing Group Ltd and the Health Foundation.
T N 2005, draft publication guidelines for quality improvement reporting debuted in Quality and Safety in Health Care. ${ }^{1}$ At that time, publications of scholarly work about health care improvement were often confusing and of limited value. Leaders in the field were working to consolidate the evidence for a science of improvement, ${ }^{2,3}$ and without guidance on how to write their findings, authors struggled to report their improvement work in a reliable and consistent way. ${ }^{4,5}$ These factors influenced the initial

Supplemental digital content is available for this article. Direct URL citation appears in the printed text and is provided in the HTML and PDF versions of this article on the journal's Web site (www.jncqjournal.com).

Correspondence: Greg Ogrinc, MD, MS, Geisel School of Medicine at Dartmouth, 1 Rope Ferry Rd, Hanover, NH 03755 (Greg.Ogrinc@va.gov).

Published abead of print: September 30, 2015

DOI: $10.1097 / N C Q .0000000000000153$ 
publication in 2008 of the Standards for QUality Improvement Reporting Excellence (SQUIRE), ${ }^{6}$ which we refer to as SQUIRE 1.0. The guidelines were developed in an effort to reduce uncertainty about the information deemed to be important in scholarly reports of health care improvement and to increase the completeness, precision, and transparency of those reports.

In the intervening years, the reach of systematic efforts to improve the quality, safety, and value of health care has grown. Health professions education worldwide now includes improvement as a standard competency. ${ }^{7-11}$ The science of the field also continues to advance through guidance on applying formal and informal theory in the development and interpretation of improvement programs ${ }^{12}$; stronger ways to identify, assess, and describe context ${ }^{13-16}$; recommendations for clearer, more complete descriptions of interventions ${ }^{17}$; and development of initial guidance on how to study an intervention. ${ }^{18}$

In this setting, we have undertaken a revision of SQUIRE 1.0. When we began, it rapidly became apparent that a wide variety of approaches had developed for improving health care, ranging from formative to experimental to evaluative. Rather than limit the revised guidelines to only a few of these, we fashioned them to be applicable across the many methods that are used. We aimed to reflect the dynamic nature of the field and support its further development. This article describes the development and content of SQUIRE 2.0 (Table).

\section{SQUIRE 2.0 DEVELOPMENTAL PATH}

We developed SQUIRE 2.0 between 2012 and 2015 in 3 overlapping phases: (1) evaluation of the initial SQUIRE guidelines, (2) early revisions, and (3) pilot testing with late revisions. We began the evaluation of SQUIRE 1.0 by collecting data to assess its clarity and usability. ${ }^{19}$ Semistructured interviews and focus groups with 29 end users of SQUIRE 1.0 revealed that many found SQUIRE 1.0 helpful in planning and doing improvement work but less so in the writing process. This issue was especially apparent in efforts to write about the cyclic, iterative process that often occurs with improvement interventions. SQUIRE 1.0 was seen by many as unnecessarily complex with too much redundancy and lacking a clear distinction between "doing improvement" and "studying the improvement." A recent independent study and editorial also documented and addressed some of these challenges. ${ }^{20,21}$

In the second phase, we convened an international advisory group of 18 experts that included editors, authors, researchers, and improvement professionals. This group met through 3 conference calls, reviewed SQUIRE 1.0 and the results of the end-user evaluation, and provided detailed feedback on successive revisions. This advisory group and additional participants attended 2 consensus conferences in 2013 and 2014 where they engaged in intensive analysis and made recommendations that further guided the revision process.

In the third phase, 44 authors used an interim draft version of the updated SQUIRE guidelines to write sections of a manuscript. Each author then provided comments on the utility and understandability of the draft guidelines, and in their submitted section, identified the portions of their writing sample that fulfilled the items of that section. ${ }^{22}$ We also obtained detailed feedback about this draft version through semistructured interviews with 11 biomedical journal editors. The data from this phase revealed areas needing further clarification and which specific items were prone to misinterpretation. Finally, a penultimate draft was e-mailed to more than 450 individuals around the world, including the advisory group, consensus meeting participants, authors, reviewers, editors, faculty in fellowship programs, and trainees. This version was also posted on the SQUIRE Web site, with an invitation for public feedback. We used the information from this process to write SQUIRE 2.0 (Table).

\section{SQUIRE 2.0}

Many publication guidelines, including CONSORT (randomized trials), STROBE (observational studies), and PRISMA (systematic 
Table. Revised SQUIRE 2.0 Publication Guidelines

\section{Text Section and Item Name}

Notes to authors

Title and Abstract

1. Title

\section{Abstract}

Introduction

3. Problem description

4. Available knowledge

5. Rationale

6. Specific aims

Methods

7. Context

8. Intervention(s)

9. Study of the intervention(s)

10. Measures

\section{Section or Item Description}

- The SQUIRE guidelines provide a framework for reporting new knowledge about how to improve health care

- The SQUIRE guidelines are intended for reports that describe system-level work to improve the quality, safety, and value of health care and used methods to establish that observed outcomes were due to the intervention(s).

- A range of approaches exists for improving health care. SQUIRE may be adapted for reporting any of these.

- Authors should consider every SQUIRE item, but it may be inappropriate or unnecessary to include every SQUIRE element in a particular manuscript.

- The SQUIRE Glossary contains definitions of many of the key words in SQUIRE.

- The Explanation and Elaboration document provides specific examples of well-written SQUIRE items and an in-depth explanation of each item.

- Please cite SQUIRE when it is used to write a manuscript.

Indicate that the manuscript concerns an initiative to improve health care (broadly defined to include the quality, safety, effectiveness, patient-centeredness, timeliness, cost, efficiency, and equity of health care)

a. Provide adequate information to aid in searching and indexing

b. Summarize all key information from various sections of the text using the abstract format of the intended publication or a structured summary such as background, local problem, methods, interventions, results, conclusions

Why did you start?

Nature and significance of the local problem

Summary of what is currently known about the problem, including relevant previous studies

Informal or formal frameworks, models, concepts, and/or theories used to explain the problem, any reasons or assumptions that were used to develop the intervention(s), and reasons why the intervention(s) was expected to work

Purpose of the project and of this report

What did you do?

Contextual elements considered important at the outset of introducing the intervention(s)

a. Description of the intervention(s) in sufficient detail that others could reproduce it

b. Specifics of the team involved in the work

a. Approach chosen for assessing the impact of the intervention(s)

b. Approach used to establish whether the observed outcomes were due to the intervention(s)

a. Measures chosen for studying processes and outcomes of the intervention(s), including rationale for choosing them, their operational definitions, and their validity and reliability

b. Description of the approach to the ongoing assessment of contextual elements that contributed to the success, failure, efficiency, and cost

c. Methods employed for assessing completeness and accuracy of data (continues) 
Table. Revised SQUIRE 2.0 Publication Guidelines (Continued)

\section{Text Section and Item Name}

11. Analysis

12. Ethical considerations

Results

13. Results

Discussion

14. Summary

15. Interpretation

16. Limitations

17. Conclusions

Other information

18. Funding

\section{Section or Item Description}

a. Qualitative and quantitative methods used to draw inferences from the data

b. Methods for understanding variation within the data, including the effects of time as a variable

Ethical aspects of implementing and studying the intervention(s) and how they were addressed, including, but not limited to, formal ethics review and potential conflict(s) of interest

What did you find?

a. Initial steps of the intervention(s) and their evolution over time (eg, time line diagram, flowchart, or table), including modifications made to the intervention during the project

b. Details of the process measures and outcome

c. Contextual elements that interacted with the intervention(s)

d. Observed associations between outcomes, interventions, and relevant contextual elements

e. Unintended consequences such as unexpected benefits, problems, failures, or costs associated with the intervention(s).

f. Details about missing data

What does it mean?

a. Key findings, including relevance to the rationale and specific aims

b. Particular strengths of the project

a. Nature of the association between the intervention(s) and the outcomes

b. Comparison of results with findings from other publications

c. Impact of the project on people and systems

d. Reasons for any differences between observed and anticipated outcomes, including the influence of context

e. Costs and strategic trade-offs, including opportunity costs

a. Limits to the generalizability of the work

b. Factors that might have limited internal validity such as confounding, bias, or imprecision in the design, methods, measurement, or analysis

c. Efforts made to minimize and adjust for limitations

a. Usefulness of the work

b. Sustainability

c. Potential for spread to other contexts

d. Implications for practice and for further study in the field

e. Suggested next steps

Sources of funding that supported this work. Role, if any, of the funding organization in the design, implementation, interpretation, and reporting

Abbreviation: SQUIRE, Standards for QUality Improvement Reporting Excellence.

reviews), focus on a particular study methodology (www.equator-network.org). In contrast, SQUIRE 2.0 is designed to apply across the many approaches used for systematically improving the quality, safety, and value of health care. Methods range from iterative changes using PDSA (Plan-Do-StudyAct) cycles in single settings to retrospective analyses of large-scale programs to multisite randomized trials. We encourage authors to apply other publication guidelinesparticularly those that focus on specific study 
methods-along with SQUIRE, as appropriate. Authors should carefully consider the relevance of each SQUIRE item but recognize that it is sometimes not necessary, nor even possible, to include each item in a particular manuscript.

SQUIRE 2.0 retains the IMRaD (Introduction, Methods, Results, and Discussion) structure. ${ }^{23}$ Although used primarily for reporting research within a spectrum of study designs, this structure expresses the underlying logic of most systematic investigations and is familiar to authors, editors, reviewers, and readers. We continue to use A. Bradford Hill's 4 fundamental questions for writing: Why did you start? What did you do? What did you find? What does it mean? ${ }^{24}$ In our evaluation of SQUIRE 1.0, novice authors found these questions to be straightforward, clear, and useful.

SQUIRE 2.0 contains 18 items but omits the multiple subitems that were a source of confusion for SQUIRE 1.0 users. ${ }^{19}$ A range of approaches exists for improving health care, and SQUIRE may be adapted for reporting any of these. As stated earlier, authors should consider every SQUIRE item, but it may be inappropriate or unnecessary to include every SQUIRE item in a particular manuscript. In addition, authors need not use items in the order in which they appear. Major changes between SQUIRE 1.0 and SQUIRE 2.0 are concentrated in 4 areas: (1) terminology, (2) theory, (3) context, and (4) studying the intervention(s).

\section{Terminology}

The elaborate detail in SQUIRE 1.0 was seen by users as a both a blessing and a curse ${ }^{19}$ : helpful in designing and executing quality improvement work but less useful in the writing process. The level of detail sometimes led to confusion about what or what not to include in a manuscript. Consequently, we made the items in SQUIRE 2.0 shorter and more direct.

A major challenge in the reporting of systematic efforts to improve health care is the multiplicity of terms used to describe the work, which is challenging for novices and experts alike. Improvement work draws on the epistemology of a variety of fields, and depending on one's field of study, the same words can carry different connotations, a particularly undesirable state of affairs. Terms such as "quality improvement," "implementation science," and "improvement science" refer to approaches that not only have many similarities but can also connote important (and often-debated) differences. Other terms such as "health care delivery science," "patient safety," and even simply "improvement" are also subject to surprising variation in interpretation. To address this problem in semantics, we created a glossary of terms used in SQUIRE 2.0 (see Supplemental Digital Content, Glossary, available at: http://links.lww. com/JNCQ/A218). The glossary provides the intended meaning of certain key terms as we have used them in SQUIRE 2.0 (Table). These definitions may be helpful in other endeavors but are not necessarily intended to be adopted for use in other contexts. Overall, we sought terms and definitions that would be useful to the largest possible audience. For example, we chose "intervention(s)" to refer to the changes that are made. We decided not to use the word "improvement" in the individual items (although it remains in the SQUIRE acronym) to encourage authors to report efforts that did not lead to changes for the better. Reporting well-done, negative studies is vital for the learning in this discipline.

\section{Theory}

SQUIRE 2.0 includes a new item titled "Rationale." Biomedical and clinical research is driven by iterative cycles of theory building and hypothesis testing. Health care improvement work has not consistently based the planning, design, and execution of its programs solidly in theory, to the detriment of the work. For this reason, SQUIRE $2.0 \mathrm{ex}$ plicitly includes an item devoted to theory, although we chose to use the broader and less technical label "Rationale," to encourage authors to be explicit in reporting formal and informal theories, models, concepts, or even hunches as to why they expected a particular intervention to work in a particular context. A plain language interpretation of 
"Rationale" might be "Why did you think this would work?" A recent narrative review of the nature of theory and its use in improvement describes the many types and applications of theory and considers pitfalls in using, and not using, theory. ${ }^{12}$

The addition of the "Rationale" item is intended to encourage clarity around assumptions about the nature of the intervention, context, and expected outcomes. The presence of a well-thought-out rationale will align with appropriate measures and with the study of the intervention; it may also be the starting point for the next round of work. The "Summary" item in the "Discussion" section encourages authors to revisit the original rationale in the light of its findings and in the larger context of similar projects.

\section{Context}

SQUIRE 2.0 accepts "context" as the key features of the environment in which the work is immersed and which are interpreted as meaningful to the success, failure, and unexpected consequences of the intervention(s), as well as the relationship of these to the stakeholders (eg, improvement team, clinicians, patients, families). ${ }^{13-16}$ Systematic efforts to improve health care should contain clear descriptions and acknowledgement of context rather than efforts to control it or explain it away. SQUIRE 1.0 included context with items in all sections of the manuscript, but context did not rise to the level of a distinct item itself. SQUIRE 2.0 recognizes context as a fundamental item in the "Methods" section, but its relevance is not limited to this section. In addition to affecting the development of the rationale and subsequent design of the intervention(s), context plays a key role in the iterations of intervention(s) and the outcomes. While it is often not simple to capture or describe context, understanding its impact on the design, implementation, measurement, and results makes it a vital contributor in identifying and reporting the factors and mechanisms responsible for the success or failure of the intervention(s).

\section{Studying the intervention(s)}

The study of the intervention is, perhaps, the most challenging item in SQUIRE. In the evaluation of SQUIRE $1.0^{19}$ and in the pilot testing, ${ }^{22}$ many were perplexed by this item and its subelements. This item was intended to encourage a more formal assessment of the intervention and its associated outcomes. In SQUIRE 2.0, this section is called, "Study of the Intervention(s)" (Table).

"Doing" an improvement project is fundamentally different from "studying" it. The primary purpose of "doing" improvement is to produce better local processes and outcomes rather than contribute to new generalizable knowledge. In contrast, the reason for "studying" the intervention is mainly to contribute to the body of knowledge about the efficacy and generalizability of efforts for improving health care. Both "doing" and "studying" are required for a deep understanding of the nature and impact of the intervention(s), as well as the possible underlying mechanisms. "Study of the Intervention(s)" focuses mainly on whether and why an intervention "works." It should align with the rationale and may include, but is not limited to, preplanned formal testing of the proposed theory that the intervention(s) actually produced the observed changes, as well as the impact of the intervention(s) on the context in which the work was done.

SQUIRE 2.0 asks authors to be as transparent, complete, and as accurate as possible about reporting "doing" and "studying" improvement work, as both aspects of the work are key to scholarly reporting. The "Summary" and "Interpretation" items in the "Discussion" section encourage authors to explain potential mechanisms by which the intervention(s) resulted (or failed to result) in change, thereby developing explanatory theories that can be subsequently tested.

\section{CONCLUSIONS}

The development of SQUIRE 2.0 consisted of a detailed analysis of SQUIRE 1.0, input 
from experts in the field, and thorough pilot testing. Many methods and philosophical approaches to improve the quality, safety, and value of health care are available. The systematic efforts to improve health care are often complex and multidimensional, and their effectiveness is inherently context dependent. SQUIRE 2.0 provides common ground on which the discoveries contributed by the various approaches can advance the field by sharing them in the published literature.

At the same time, we recognize that simply publishing SQUIRE 2.0 will not effect this change; additional efforts and resources are required. For example, we have created an explanation and elaboration (E\&E) document (D. Goodman, et al, written communication, 2015) to accompany this article. For each item in SQUIRE 2.0, the E\&E provides
1 or more examples from the published literature and a commentary on how the example(s) meets or does not meet the item's standards; this information brings the content of each item to life. The SQUIRE Web site (www.squire-statement.org) contains a number of resources in addition to the guidelines themselves, including interactive E\&E pages and video commentaries. The Web site supports an emerging online community for the continuous use, conversation about, and evaluation of the guidelines.

Writing about improvement can be challenging. Sharing successes, failures, and developments through scholarly literature is an essential component of the complex work required to improve health care services for patients, professionals, and the public.

\section{REFERENCES}

1. Davidoff F, Batalden P. Toward stronger evidence on quality improvement. Draft publication guidelines: the beginning of a consensus project. Qual Saf Health Care. 2005;14(5):319-325.

2. DeVinney B, ed. Expanding Research and Evaluation Designs to Improve the Science Base for Health Care and Public Health Quality Improvement Symposium. Conference Summary. Rockville, MD: Agency for Healthcare Research and Quality; March 2009.

3. Shojania KG, Grimshaw JM. Evidence-based quality improvement: the state of the science. Health Aff. 2005;24(1):138-150.

4. Grol RP, Bosch MC, Hulscher ME, Eccles MP, Wensing $M$. Planning and studying improvement in patient care: the use of theoretical perspectives. Milbank $Q$. 2007;85(1):93-138.

5. Rubenstein LV, Hempel S, Farmer MM, et al. Finding order in heterogeneity: types of quality-improvement intervention publications. Qual Saf Health Care. 2008;17(6):403-408.

6. Davidoff F, Batalden P, Stevens D, Ogrinc G, Mooney S. Publication guidelines for quality improvement in health care: evolution of the SQUIRE project. Qual Saf Health Care. 2008;17(suppl 1):i3-i9.

7. Batalden P, Leach D, Swing S, Dreyfus H, Dreyfus S. General competencies and accreditation in graduate medical education. Health Aff. 2002;21(5):103-111.

8. Interprofessional Education Collaborative Expert Panel. Core Competencies for Interprofessional Col- laborative Practice: Report of an Expert Panel. Washington, DC: Interprofessional Education Collaborative; 2011.

9. Association of American Medical Colleges. Teaching for Quality. https://www.aamc.org/initiatives/ cei/te4q. Published 2013. Accessed June 21, 2013.

10. Cronenwett L, Sherwood G, Barnsteiner J, et al. Quality and safety education for nurses. Nurs Outlook. 2007;55(3):122-131.

11. Nasca TJ, Philibert I, Brigham T, Flynn TC. The Next GME Accreditation System-rationale and benefits. $N$ Engl J Med. 2012;366(11):1051-1056.

12. Davidoff F, Dixon-Woods M, Leviton L, Michie S. Demystifying theory and its use in improvement [published online ahead of print January 23, 2015]. BMJ Qual Saf. doi:10.1136/bmjqs-2014-003627.

13. Bate P, Robert G, Fulop N, Ovretveit J, Dixon-Woods M. Perspectives on Context. London, England: The Health Foundation; 2014.

14. Kaplan HC, Provost LP, Froehle CM, Margolis PA. The Model for Understanding Success in Quality (MUSIQ): building a theory of context in healthcare quality improvement. BMJ Qual Saf. 2012;21(1): 13-20.

15. Øvretveit J. Understanding the conditions for improvement: research to discover which context influences affect improvement success. BMJ Qual Saf. 2011;20(suppl 1):i18-i23.

16. Taylor SL, Dy S, Foy R, et al. What context features might be important determinants of the effectiveness 
of patient safety practice interventions? BMJ Qual Saf. 2011;20(7):611-617.

17. Hoffmann TC, Glasziou PP, Boutron I, et al. Better reporting of interventions: Template for Intervention Description and Replication (TIDieR) checklist and guide. BMJ. 2014;348:1-12.

18. Portela MC, Pronovost PJ, Woodcock T, Carter P, Dixon-Woods M. How to study improvement interventions: a brief overview of possible study types. BMJ Qual Saf. 2015;24(5):325-336.

19. Davies L, Batalden P, Davidoff F, Stevens D, Ogrinc G. The SQUIRE guidelines: an evaluation from the field, five years post release [published online ahead of print June 18, 2015]. BMJ Qual Saf. doi:bmjqs2015-004116.

20. Howell V, Schwartz AE, O'Leary JD, Mc Donnell C. The effect of the SQUIRE (Standards of QUality Improvement Reporting Excellence) guidelines on reporting standards in the quality improvement literature: a before-and-after study [published online ahead of print February 12, 2015]. BMJ Qual Saf. doi:bmjqs2014-003737.

21. Stevens D. SQUIRE and the evolving science of healthcare improvement [published online ahead of print March 27, 2015]. BMJ Qual Saf. 2015;24:349-351. doi:10.1136/bmjqs-2015-004158.

22. Davies L, Donnelly K, Goodman D, Ogrinc G. Findings from a novel approach to publication guideline revision: user road testing of a draft version of SQUIRE 2.0 [published online ahead of print August 11, 2015]. BMJ Qual Saf. doi:10.1136/bmjqs-2015004117.

23. Day RA. The origins of the scientific paper: the IMRaD format. J Am Med Writers Assoc. 1989;4(2):16-18.

24. Huth E. Writing and Publishing in Medicine. 3rd ed. Baltimore, MD: Williams \& Wilkins; 1999. 